\section{IDDF2019-ABS-0278 FUSOBACTERIUM NUCLEATUM PROMOTES MALIGNANT PROGRESSION OF COLORECTAL CANCER BY ACTIVATING NF- KB/NCOA7-AS1/IKK POSITIVE FEEDBACK LOOP}

${ }^{1}$ Changsheng Yan*, ${ }^{2}$ Xiang Zhang. 'Zhongshan Hospital Xiamen University, China; ${ }^{2} T$ The Chinese University of Hong Kong, Hong Kong

\subsection{6/gutjnl-2019-IDDFabstracts.57}

Background As Fusobacterium nucleatum has been demonstrated to play an important role in the progression of colorectal cancer. To elucidate the potential mechanism of Fusobacterium nucleatum in the malignant progression of colorectal cancer is of big importance.

Methods The dataset in the colorectal cancer group was analyzed by edger function and limma function. QRT-PCR was conducted to detect gene and bacterial expressive abundances. Regulatory effects of Fusobacterium nucleatum on cellular behaviors of colorectal cancers were evaluated through CCK8 , wound healing assay and flow cytometry. The binding relationship and interaction between NF-kB and NCOA7-AS1 were verified through dual-luciferase reporter gene assay, ChIP and functional experiments. Moreover, proteins that bind to NCOA7-AS1 were determined by RNA-Pull down, mass spectrometry and RIP.

Results LncRNA NCOA7-AS1 is upregulated in colorectal cancer infected with Fusobacterium nucleatum. Correlation analysis showed that the abundance of Fusobacterium nucleatum was positively correlated with NCOA7-AS1 level. Fusobacterium nucleatum could accelerate the malignant phenotypes of colorectal cancer, which were partially reversed by the silence of NCOA7-AS1. NF-kB was verified to bind to the NCOA7-AS1 promoter region to upregulate NCOA7-AS1. Furthermore, NCOA7-AS1 was identified to bind to IKK and developed positive feedback to activate the NF-KB pathway, thereby promoting the progression of colorectal cancer.

Conclusions Fusobacterium nucleatum activates the positive feedback loop NF-kB/NCOA7-AS1/IKK, thereby accelerating the malignant progression of colorectal cancer.

\section{IDDF2019-ABS-0287 TUMOR-ASSOCIATED MACROPHAGES OF THE M2 PHENOTYPE ENDORSE COLORECTAL CANCER METASTASIS}

Wangxiong Hu*. Cancer Institute (Key Laboratory of Cancer Prevention and Intervention, China National Ministry of Education), The Second Affiliated Hospital, Zhejiang University School of Medicine, China

\subsection{6/gutjnl-2019-IDDFabstracts.58}

Background Metastasis is the major cause of cancer. Previously much effort has been made in identifying the metastasis-specific highly or low expressed genes, often with little success. We thus postulate that tumor immune microenvironment (TIME) disorder is the fundamental reason associated with cancer progression. Here, we try to determine the candidates associated with cancer progression in colorectal cancer (CRC). Methods CRC mRNA expression data sets were obtained from The Cancer Genome Atlas (TCGA) and Gene Expression
Omnibus (GEO), respectively. CIBERSORT was used to decipher the proportions of 22 tumor-infiltrating lymphocytes (TILs). TILs that correlated with patient survival time in the multivariate Cox regression analysis were determined using the least absolute shrinkage and selection operator (LASSO) method. Survival differences between good- and poor-prognosis based on the prognostic index (PI) were tested by the Kaplan-Meier method and analyzed with the log-rank test with functions survfit and survdiff in the survival package for R. A $P$ value $<0.05$ was considered significant.

Results Tumor-associated macrophages (TAMs) of the M2 phenotype was significantly enriched in stage IV CRCs than in other stages. CRC patients could be separated into two groups with high or low PI based on the proportion of mast cell and M2 macrophages. In addition, a negative correlation of M2 macrophages and CD8 T cell was observed.

Conclusions Targeting pro-metastatic immune cells such as macrophages (e.g., transform into M1-like macrophages) may be a possible therapeutic strategy for CRC.

\section{IDDF2019-ABS-0288 FATTY ACID CATABOLISM IMPACTS THE SENSITIVITY OF GASTROINTESTINAL CANCERS TO PLATINUM-BASED CHEMOTHERAPY}

Ying-nan Wang*. Sun Yat-sen University Cancer Center, China

\subsection{6/gutjnl-2019-IDDFabstracts.59}

Background Carnitine palmitoyltransferases (CPTs) are capable of transporting fatty acids (FAs) into mitochondria to synthesize cardiolipin and initiate $\beta$-oxidation. The aim of this study was to identify the inhibition of CPT-mediated FA catabolism on oxaliplatin sensitivity and its therapeutic potential in gastrointestinal cancers.

Methods We investigated oxaliplatin-induced metabolic alterations, disease control rates (DCRs) using gastric cancer (GC) and colorectal cancer (CRC) cells as well as CRC tissues from patients treated with FOLFOX or XELOX regimens $(n=82)$. The effects of CPT inhibitor perhexiline on the growth of GC and CRC were tested in vitro and in vivo.

Results CPT1B and CPT2 were significantly upregulated when GC and CRC cells were treated with oxaliplatin, and increased CPT1B and CPT2 expression correlated with a worse DCR in patients receiving FOLFOX or XELOX. Suppression of CPT2 or delivering perhexiline inhibited the progression of GC and CRC cells by inducing apoptosis, which was due to the loss of cardiolipin, suppression of $\beta$ oxidation, destruction of mitochondrial membrane integrity and reduction in ATP production. This process enhanced the antitumor effect of oxaliplatin in vitro and in vivo. Especially, perhexiline treatment significantly suppressed the progression of GC and CRC in patient-derived xenograft (PDX) models, which was closely associated with CPT2 expression.

Conclusions Activated expression of FA catabolism is related to the chemoresistance of patients with GC and CRC. Inhibition of CPT cooperates with oxaliplatin to suppress their growth, as CPT1B and CPT2 are potential biomarkers for platinum-based chemotherapy in GC and CRC. 